\title{
A Note on Fuzzy Soft Ditopological Spaces
}

\author{
Tuğbahan ŞİMŞEKLER DİZMAN* (D)
}

Gaziantep University, Gaziantep Education Faculty, Gaziantep, Turkiye

Geliş / Received: 18/10/2018, Kabul / Accepted: 11/07/2019

\begin{abstract}
In this study, we define fuzzy soft ditopological spaces as the generalization of soft ditopological spaces defined by Simsekler et. al. (2016). Fuzzy soft ditopological space is a combination of fuzzy soft topological and fuzzy soft cotopological spaces which are defined by two independent structures fuzzy soft open and fuzzy soft closed sets. Also we give fuzzy soft $\tau$-continuity in fuzzy soft topology, fuzzy soft $\kappa$-continuity in fuzzy soft cotopology and by using these two types of continuity we finally define the fuzzy soft continuity in a fuzzy soft ditopology.
\end{abstract}

Keywords: Fuzzy soft sets, fuzzy soft ditopology, fuzzy soft continuity.

\section{Bulanık Esnek Ditopolojiler Üzerine Bir Çalışma}

\section{Öz}

Bu çalışmada, Simsekler Dizman ve arkadaşları tarafından (2016) tanımlanan esnek ditopolojik uzayların bir genelleştirilmesi olan bulanık esnek ditopolojik uzaylar tanımlanmıştır. Bulanık esnek ditopolojik uzaylar birbirinden bağımsız olarak tanımladığımız bulanık esnek açık ve bulanık esnek kapalı kümeler kullanılarak tanımlanmış bulanık esnek topolojiler ve bulanık esnek kotopolojilerin bir kombinasyonudur. Ayrıca bulanık esnek topolojilerde tanımladığımız $\tau$-süreklilik ve bulanık esnek kotopolojilerde $\kappa$-sürekiliği kullanarak bulanık esnek ditopolojilerde sürekliliği verdik.

Anahtar Kelimeler: Bulanık esnek kümeler, bulanık esnek ditopoloji, bulanık esnek süreklilik

\section{Introduction}

Since the real life problems in several areas are more objective, solving these problems by classical mathematics rules is not appropriate many times. Fuzzy set theory defined by Zadeh (1965) gives us an opportunity to define a set and its elements by a different way than the well-known approach 'Black or White' and presents us a new angle to sight the connections between the set and its elements. The main opinion of the theory is defining a fuzzy set by the fuzzy membership function and hence state that which "degree", an element pertion to a fuzzy set. Chang (1968) was first investigated fuzzy set topology. The soft set was described in 1999 by Molodtsov (1999) as a new approach for uncertainity and the theory is based on defining a soft set by a parameter set of objects in the universe with a mapping. It is easily seen that fuzzy and soft sets are interdependent (Aktas and Cagman, 2007). Maji et al. (2001) first studied the hybrid 
model of fuzzy and soft sets and defined fuzzy soft set (briefly fs-set) as a new type of vague sets. Ahmad and Kharal (2012) contributed to the fuzzy soft theory and described the concept of a mapping between fs-sets. Aktas and Cagman (2007) defined the notion of soft groups and some of their properties. Feng et al. (2008) studied the soft semirings and investigated several related properties. Nazmul and Samanta (2010) contributed the algebraic structures of soft sets. Shabir and Naz (2011) described the soft topological spaces and separation axioms of soft topological spaces. Tanay and Kandemir (2011) searched the topology of fs-sets.

Soft and fs-sets and the structures on these sets were observed by several researchers (Ahmad and Hussain, 2012, Atmaca and Zorlutuna, 2014, Aygunoglu and Aygun, 2011, Aygunoglu et. al., 2014, Bera et.al., 2017, Cagman and Aktas, 2011, Cagman et. al., 2011, Karaaslan et. al., 2012, Karasalan 2016, Karaslan et. al., 2013, Min, 2011, Ozturk, 2016, Pazar and Aygun, 2012, Roy and Samanta, 2012, Roy and Samanta, 2013, Simsekler ve Yuksel, 2013, Zorlutuna et. al., 2012).

Ditopology was first given by L.M. Brown and investigated by L.M. Brown and coauthors (1998). Actually ditopology is connected with the notion of the bitopology described by J.L. Kelly (1963). The ditopology is based on two structures, openness and closedness, which are defined as independent concepts from each other.

In this paper, we interpret the parameters $\mathrm{E}$ and $A$, which are used in the definition of fssets, different from other papers written on fstopology. We assume that if a parameter does not belong to $\mathrm{A}$, the value for this parameter is not defined. Considering fs-sets by this idea makes differences while defining the fs- topologies. This way leads to define fs-open sets and fs-closed sets as independent structures. We can summarize our study in the following way. First, we define fs-topological spaces by fs-open sets and we investigate the properties of this space. Then we define fscotopological spaces by using fs-closed sets. We define the concept of fs-remote neighborhood of a fs-point and observe the properties of fs-cotopological spaces. Finally, we define the fs-ditopological spaces by combining the fs-topology and cotopology and sum up the results of this paper.

\section{Preliminaries}

Throughout the paper $U$ will denote the universe and $\mathrm{E}$ will denote the parameter set and $I^{U}$ will denote the all fuzzy sets on $\mathrm{U}$. Let $\mathrm{A}$ and $\mathrm{B}$ be nonempty sets of $\mathrm{E}$.

In this section we give the main definitions of fs-set theory which can be found several papers we cited in introduction. As we state in the previous section our Fuzzy soft set definition is different from this works in the meaning of parameter set.

Definition 1. $f_{A}$ is called the fuzzy soft set (briefly fs-set over) $\mathrm{U}$ if $f_{A}: A \rightarrow I^{U}$ is a mapping defined by $f_{A}(e)=\mu_{f_{A}}{ }^{e}$ where $\mu_{f_{A}}{ }^{e}(u) \neq 0$ for each $u \in U$ otherwise i.e, $e \notin A, f_{A}(e)$ will not be considered.

Definition 2. The complement of a fs-set $f_{A}$ is a fs-set denoted by $f_{A}{ }^{c}$ where $f_{A}{ }^{c}: A \rightarrow I^{U}$ is a mapping defined by $\mu_{f_{A}}^{e}(u)=1-\mu_{f_{A}}^{e}(u)$ for all $e \in A, u \in U$.

Definition 3. Let $f_{A}$ and $g_{B}$ be two fs-sets over U. $f_{A}$ is said to be a fs-subset of $g_{B}$ if $A \subseteq B$ and $\mu_{f_{A}}{ }^{e}(u) \leq \mu_{g_{B}}{ }^{e}(u)$ for each $e \in A$ and $u \in U$.

Definition 4. Let $f_{A}$ and $g_{B}$ be two fs-sets over U. The intersection of $f_{A}$ and $g_{B}$ denoted by 
$f_{A} \sqcap g_{B}$ is a fs-set $h_{C}$ where $C=A \cap B$ and is defined by

$$
{\mu_{H_{C}}}^{e}(u)=\min \left\{{\mu_{f_{A}}}^{e}(u), \mu_{g_{B}}{ }^{e}(u)\right\}, \forall e \in C, \forall u \in U .
$$

Definition 5. Let $f_{A}$ and $g_{B}$ be two fs-sets $f_{A} \sqcup g_{B}$ is a fs-set $h_{C}$ where $C=A \cup B$ and over $\mathrm{U}$. The union of $f_{A}$ and $g_{B}$ denoted by is defined by

$$
\mu_{H_{C}}{ }^{e}(u)=\left\{\begin{array}{c}
\mu_{f_{A}}{ }^{e}(u), \text { if } e \in A-B \\
\mu_{g_{B}}{ }^{e}(u), \text { if } e \in B-A \\
\max \left\{\mu_{f_{A}}{ }^{e}(u), \mu_{g_{B}}{ }^{e}(u)\right\}, \text { if } e \in A \cap B
\end{array}, \forall u \in U .\right.
$$

Definition 6. The fs-set $f_{A}$ over $\mathrm{U}$ is defined to be null fs-set and is denoted by $\Phi$ where $f_{A}(e)=0^{-}, \forall e \in A, \forall u \in U$.

Definition 7. The fs-set $f_{A}$ over $U$ is defined to be absolute fs-set and is denoted by $U_{E}^{\sim}$ where $f_{A}(e)=I^{-}, \forall e \in A, \forall u \in U$.

Theorem 1. Let $f_{A}$ and $g_{B}$ be two fs-sets over $\mathrm{U}$. Then the followings hold:

i) $\quad\left(f_{A} \sqcap g_{B}\right)^{c} \sqsubseteq f_{A}{ }^{c} \sqcup g_{B}{ }^{c}$.

ii) $\quad f_{A}{ }^{c} \sqcap g_{B}{ }^{c} \sqsubseteq\left(f_{A} \sqcup g_{B}\right)^{c}$.

Proof. i) Let $\left(f_{A} \sqcap g_{B}\right)^{c}=h_{C}, C=A \cap B$. Then, $\mu_{h_{C}}^{e}(u)=\max \left\{\mu_{f_{A}^{c}}^{e}(u), \mu_{g_{B}^{c}}^{e}(u)\right\}$, for all $e \in A \cap B$ and for all $u \in U$. On the other hand, let $f_{A}{ }^{c} \sqcup g_{B}{ }^{c}=k_{M}, M=A \cup B$.

$$
\begin{aligned}
& \mu_{k_{M}}^{e}(u) \\
& =\left\{\begin{array}{c}
\mu_{f_{A}^{c}}^{e}(u), e \in A-B \\
\mu_{g_{B}^{c}}^{e}(u), e \in B-A \\
\max \left\{\mu_{f_{A}^{c}}^{e}(u), \mu_{g_{B}^{c}}^{e}(u)\right\}, e \in A \cap B
\end{array}\right.
\end{aligned}
$$

Hence the proof is completed.

$$
f_{A}{ }^{c} \sqcup g_{B}{ }^{c}=\left\{e_{1}=\left\{u_{1}^{0.7}, u_{2}^{0.6}, u_{3}^{1}\right\}, e_{2}=\left\{u_{1}^{1}, u_{2}^{0.5}, u_{3}^{0.4}\right\}, e_{3}=\left\{u_{1}^{1}, u_{2}^{1}, u_{3}^{0.8}\right\}\right\} \text {. }
$$

On the other side,

$$
\begin{aligned}
& \left(f_{A} \sqcap g_{B}\right)^{c}=\left\{e_{1}=\left\{u_{1}^{0.7}, u_{2}^{0.6}, u_{3}^{1}\right\}\right\} . \quad \text { subset of }\left(f_{A} \sqcap g_{B}\right)^{c} . \\
& \text { ii) }\left(f_{A} \sqcup g_{B}\right)^{c}=\left\{e_{1}=\left\{u_{1}^{0.5}, u_{2}^{0.3}, u_{3}^{0.4}\right\}, e_{2}=\left\{u_{1}^{1}, u_{2}^{0.5}, u_{3}^{0.4}\right\}, e_{3}=\left\{u_{1}^{1}, u_{2}^{1}, u_{3}^{0.8}\right\}\right\} .
\end{aligned}
$$$$
\text { It can be easily seen that } f_{A}{ }^{c} \sqcup g_{B}{ }^{c} \text { is not a }
$$

ii) Let $\left(f_{A} \sqcup g_{B}\right)^{c}=h_{C}$. Then

$$
\begin{aligned}
& \mu_{h_{C}}^{e}(u) \\
& =\left\{\begin{array}{c}
\mu_{f_{A}^{c}}^{e}(u), e \in A-B \\
\mu_{g_{B}^{c}}^{e}(u), e \in B-A \\
\min \left\{\mu_{f_{A}^{c}}^{e}(u), \mu_{g_{B}^{c}}^{e}(u)\right\}, e \in A \cap B
\end{array}\right.
\end{aligned}
$$

On the other hand let

$f_{A}{ }^{c} \sqcap g_{B}{ }^{c}=k_{M}, M=A \cap B$. Then,

$\mu_{k_{M}}^{e}(u)=\min \left\{\mu_{f_{A}^{c}}^{e}(u), \mu_{g_{B}^{c}}^{e}(u)\right\}, e \in A \cap B$.

Hence the proof is completed.

Remark 1. The inverse inclusions do not hold generally.

Example 1. Let $U=\left\{u_{1}, u_{2}, u_{3}\right\}, \quad E=$ $\left\{e_{1}, e_{2}, e_{3}\right\}, A=\left\{e_{1}, e_{2}\right\}, B=\left\{e_{1}, e_{3}\right\}$. Let $f_{A}, g_{B}$ be two fs-sets as defined by,

$$
\begin{aligned}
& f_{A}=\left\{e_{1}=\left\{u_{1}^{0.5}, u_{2}^{0.7}\right\}, e_{2}=\left\{u_{2}^{0.5}, u_{3}^{0.6}\right\}\right\}, \\
& g_{B}=\left\{e_{1}=\left\{u_{1}^{0.3}, u_{2}^{0.4}, u_{3}^{0.6}\right\}, e_{3}=\left\{u_{3}^{0.2}\right\}\right\} .
\end{aligned}
$$

i) We obtain the union of $f_{A}{ }^{c}, g_{B}{ }^{c}$.
Now we obtain the intersection of $f_{A}{ }^{c}, g_{B}{ }^{c}$.

$$
f_{A}{ }^{c} \sqcap g_{B}{ }^{c}=\left\{e_{1}=\left\{u_{1}^{0.5}, u_{2}^{0.3}, u_{3}^{0.4}\right\}\right\} \text {. }
$$

It can be easily seen that $\left(f_{A} \sqcup g_{B}\right)^{c}$ is not a subset of $f_{A}{ }^{c} \sqcap g_{B}{ }^{c}$.

\section{Fuzzy Soft Topological Spaces}

Fs-topological spaces were defined and searched in several papers. (Aygunoglu et. al 2014, Atmaca and Zorlutuna 2014, Pazar and Aygun 2012, Roy and Samanta 2012, Roy and Samanta 2013, Simsekler and Yuksel 2013). In this work the interpretation of fs-sets is 
different from the published papers and this leads different results while defining fstopological spaces and the properties of this spaces. Also in this section we use only fsopenness as an independent structure from closedness.

Definition 7. Let $U$ be the universe and $E$ be a parameter set. The fs-topological space is a pair $\left(U_{E}^{\sim}, \tau_{f}\right)$ where $\tau_{f}$ is a family of fs-sets over U satisfying the following conditions:

i) $\Phi, U_{E}^{\sim} \in \tau_{f}$,

ii) If $f_{A}, g_{B} \in \tau_{f}$ then $f_{A} \sqcap g_{B} \in \tau_{f}$,

iii) If $f_{A_{i}} \in \tau_{f}$ for all $i \in I$ then $\sqcup_{i \in I} f_{A_{i}} \in \tau_{f}$.

$\tau_{f}$ is called a fuzzy soft topology (briefly fstopology) of fs-sets over $\mathrm{U}$. The pair $\left(U_{E}^{\sim}, \tau_{f}\right)$ is called fs-topological space. The elements of $\tau$ are defined to be fs-open sets.

Definition 8. (Atmaca and Zorlutuna, 2013) The fs-set $f_{A}$ is called a fs-point and denoted by $e_{x}^{\lambda}$ if there exists the parameter $e$ and the fuzzy point $x_{\lambda}$ such that $f_{A}^{e}(x)=\lambda$ where $x \in$ $U$ and $\lambda \in(0,1]$.

Definition 9. (Atmaca and Zorlutuna,2013) $e_{x}^{\lambda}$ is called an element of $f_{A}$ denoted by $e_{x}^{\lambda} \in^{\sim} f_{A}$ if $\lambda \leq \mu_{f_{A}}{ }^{e}(x)$.

Lemma 1. If $e_{x}^{\lambda} \sqcap f_{A}=\Phi$ then $e_{x}^{\lambda} \notin^{\sim} f_{A}$.

Proof. Let $\quad e_{x}^{\lambda} \sqcap f_{A}=\Phi$. Then $\min \left\{\lambda, \mu_{f_{A}}{ }^{e}(x)\right\}=0$. This shows that $\mu_{f_{A}}{ }^{e}(x)=0$ and hence $e_{x}^{\lambda} \notin^{\sim} f_{A}$.

Remark 2. The inverse inclusion of theorem does not satisfy generally.

Example 2. Let $U=\{x, y\}, E=\{k, l, m\}$. Let $f_{A}=\left\{l=\left\{x_{0.7}, y_{0.6}\right\}, m=\left\{x_{0.3}, y_{0.2}\right\}\right\} \quad$ be a fs-set over $\mathrm{U}$ and $m_{y}^{0.6}$ be a fs-point. It can be easily shown that $m_{y}^{0.6} \notin^{\sim} f_{A}$ but $m_{y}^{0.6} \sqcap f_{A}=$ $\left\{m=\left\{y_{0.2}\right\}\right.$.

Definition 10. Let $\left(U_{E}^{\sim}, \tau_{f}\right)$ be a fstopological space, $e_{x}^{\lambda}$ be a fs-point and $f_{A}$ be a fs-set. $f_{A}$ is called fs- $\tau$-neighborhood of $e_{x}^{\lambda}$ if there exists a fs-open set $g_{B}$ such that $e_{x}^{\lambda} \in^{\sim} g_{B} \sqsubseteq f_{A}$. The family of all fsneighborhoods of $e_{x}^{\lambda}$ is denoted by $\aleph\left(e_{x}^{\lambda}\right)$.

Theorem 2. Let $\left(U_{E}^{\sim}, \tau_{f}\right)$ be a fs-topological space, $e_{x}^{\lambda}$ be a fs-point and $f_{A}, g_{B}$ be two fssets. The followings hold for $\aleph\left(e_{x}^{\lambda}\right)$ :

i) If $f_{A} \in \aleph\left(e_{x}^{\lambda}\right)$ then $e_{x}^{\lambda} \in f^{\sim}$.

ii) If $f_{A}, g_{B} \in \aleph\left(e_{x}^{\lambda}\right)$ then $f_{A} \sqcap g_{B} \in$ $\aleph\left(e_{x}^{\lambda}\right)$.

iii) If $f_{A} \in \aleph\left(e_{x}^{\lambda}\right)$ and $f_{A} \sqsubseteq g_{B}$ then $g_{B} \in$ $\aleph\left(e_{x}^{\lambda}\right)$.

Proof. The proof can be done similarly to the analogous statements in Simsekler and Yuksel (2013).

Definition 11. Let $\left(U_{E}^{\sim}, \tau_{f}\right)$ be a fstopological space, $e_{x}^{\lambda}$ be a fs-point and $f_{A}$ be a fs-set $e_{x}^{\lambda}$ is called fs-interior point of $f_{A}$ if there exists a fs-open set $g_{B}$ such that $e_{x}^{\lambda} \in^{\sim} g_{B} \sqsubseteq f_{A}$.

$\operatorname{int} f_{A}=\bigsqcup_{i \in I}\left\{g_{B_{i}} \sqsubseteq U_{E}^{\sim}: g_{B_{i}} \in \tau_{f}, g_{B_{i}} \sqsubseteq f_{A}, i\right.$ $\in I\}$

Theorem 3. Let $\left(U_{E}^{\sim}, \tau_{f}\right)$ be a fs-topological space and $f_{A}$ be a fs-set. Then the followings hold:

i) $\quad \operatorname{int} f_{A} \sqsubseteq f_{A}$.

ii) $\operatorname{int} f_{A}$ is a fs-open set.

iii) $i n t f_{A}$ is the biggest fs-open set contained in $f_{A}$.

iv) $f_{A}$ is a fs-open set iff $\operatorname{int} f_{A}=f_{A}$. 
Proof. The proof can be done similarly to the analogous statements in Simsekler and Yuksel (2013).

Definition 12. (Atmaca and Zorlutuna, 2013) $e_{x}^{\lambda}$ is called quasi with $f_{A}$ and denoted by $e_{x}^{\lambda} q f_{A}$ if $\lambda+\mu_{f_{A}}^{e}(x)>1$.

Definition 13. (Atmaca and Zorlutuna, 2013) $f_{A}$ is called quasi with $g_{B}$ and denoted by $f_{A} q g_{B}$ if there exists $e \in A \cap B, u \in U$ such that $\mu_{f_{A}}^{e}(u)+\mu_{g_{B}}^{e}(u)>1$.

Definition 14. (Atmaca and Zorlutuna, 2013) Let $\left(U_{E}^{\tilde{}}, \tau_{f}\right)$ be a fs-topological space, $e_{x}^{\lambda}$ be a fs-point and $v_{A}, w_{B}$ be fs-sets. $v_{A}$ is called a fsQ-neighborhood of $e_{x}^{\lambda}$ if there exists $w_{B} \in \tau_{f}$ such that $e_{x}^{\lambda} q w_{B} \subseteq v_{A}$. The all fs-Qneighborhoods of $e_{x}^{\lambda}$ is denoted by $\mathrm{QN}\left(e_{x}^{\lambda}\right)$.

Theorem 4. Let $\left(U_{E}^{\sim}, \tau_{f}\right)$ be a fs-topological space, $e_{x}^{\lambda}$ be a fs-point and $v_{A}, w_{B}$ be fs-sets. The followings hold for $\mathrm{QN}\left(e_{x}^{\lambda}\right)$ : ii) If $v_{A}, w_{B} \in \mathrm{QN}\left(e_{x}^{\lambda}\right)$ then $v_{A} \sqcap w_{B} \in$ $\mathrm{QN}\left(e_{x}^{\lambda}\right)$.

iii) If $v_{A} \in \mathrm{QN}\left(e_{x}^{\lambda}\right)$ and $v_{A} \sqsubseteq w_{B}$ then $w_{B} \in \mathrm{QN}\left(e_{x}^{\lambda}\right)$.

Proof. The proof can be done similarly to the analogous statements in Simsekler and Yuksel (2013).

Proposition 1. (Atmaca and Zorlutuna, 2013) Let $f_{A}, g_{B}$ be two fs-sets. If $f_{A} \subseteq g_{B}$ then $f_{A}$ is not quasi coincident with $g_{B}^{c}$.

Definition 15. (Pazar and Aygun, 2012) Let $\mathrm{U}, \mathrm{V}$ be universe sets, $\mathrm{E}$ and $\mathrm{P}$ the paramer sets, FS $(\mathrm{U}, \mathrm{E})$ and $\mathrm{FS}(\mathrm{V}, \mathrm{P})$ be the families of fs-sets over $\mathrm{U}$ and $\mathrm{V}$ respectively. Let $\varphi: U \rightarrow$ $V, \psi: E \rightarrow P$ be mappings. Then the pair $(\varphi, \psi)$ is called fs-mapping and is denoted by $\varphi_{\psi}=(\varphi, \psi): F S(U, E) \rightarrow F S(V, P)$.

Let $f_{A}$ be a fs-set of FS(U,E). The image of $f_{A}$ under the fs-mapping $\varphi_{\psi}$ is a fs-set of FS(V,P) and defined by:

i) If $v_{A} \in \mathrm{QN}\left(e_{x}^{\lambda}\right)$ then $e_{x}^{\lambda} q v_{A}$.

$$
\varphi_{\psi}\left(f_{A}\right)^{p}(v)=\left\{\begin{array}{l}
\bigvee_{\varphi(u)=v} \bigvee_{\psi(e)=p} f_{A}^{e}(u), \text { if } u \in \varphi^{-1}(v), \\
0^{-}, \text {otherwise }
\end{array}\right.
$$

or $\forall p \in \psi(e)$ and $\forall v \in V$.

Let $g_{B}$ be a fs-set of FS(V,P). The preimage of $g_{B}$ under the fs-mapping $\varphi_{\psi}$ is a fs-set of $\mathrm{FS}(\mathrm{U}, \mathrm{E})$ and defined by:

$$
\varphi_{\psi}{ }^{-1}\left(g_{B}\right)^{e}(u)=\left(g_{B}\right)^{\psi(e)}(\varphi(u))
$$

If $\varphi, \psi$ are injective then $\varphi_{\psi}$ is injective, if $\varphi, \psi$ are surjective then $\varphi_{\psi}$ is surjective.

Definition 16. Let $\left(U_{E}^{\sim}, \tau_{f}\right),\left(V_{P}^{\sim}, \tau_{f}^{*}\right)$ be two fs-topological spaces. $\varphi: U \rightarrow V, \psi: E \rightarrow P$ be mappings and $e_{x}^{\lambda}$ be a fs-point. $\varphi_{\psi}=$ $(\varphi, \psi): F S(U, E) \rightarrow F S(V, P)$ is called fs- $\tau$ - continuous at $e_{x}^{\lambda}$ if for any $\tau$ - neighborhood $g_{B}$ of $\varphi_{\psi}\left(e_{x}^{\lambda}\right)$, there exists a fs- $\tau$ neighborhood $f_{A}$ of $e_{x}^{\lambda}$ such that $\varphi_{\psi}\left(f_{A}\right)$ 드 $g_{B}$.

Theorem 5. Let $\left(U_{E}^{\sim}, \tau_{f}\right),\left(V_{P}^{\sim}, \tau_{f}^{*}\right)$ be two fstopological spaces. $\varphi: U \rightarrow V, \psi: E \rightarrow P$ be mappings and $e_{x}^{\lambda}$ be a fs-point. Then the followings are equivalent:

i) $\varphi_{\psi}=(\varphi, \psi): F S(U, E) \rightarrow F S(V, P)$ is fs$\tau$-continuous mapping at $e_{x}^{\lambda}$. 
ii) For any fs- $\tau$ - neighborhood $g_{B}$ of $\varphi_{\psi}\left(e_{x}^{\lambda}\right)$ there exists a fs- $\tau$ - neighborhood $f_{A}$ of $e_{x}^{\lambda}$ such that $f_{A} \sqsubseteq \varphi_{\psi}^{-1}\left(g_{B}\right)$.

iii.) The inverse image of every fs- $\tau$ neighborhood of $\varphi_{\psi}\left(e_{x}^{\lambda}\right)$ is a fs- $\tau$ neighborhood of $e_{x}^{\lambda}$.
Proof. The proof can be done similarly to the analogous statements in Atmaca and Zorlutuna (2013).

Example 2. Let $U=\{x, y, z\}, V=\{a, b, c\}$ be the universe sets, $E=\left\{e_{1}, e_{2}\right\}, P=\left\{p_{1}, p_{2}\right\}$ be the parameter sets, $\left(U_{E}^{\sim}, \tau_{f}\right),\left(V_{P}^{\sim}, \tau_{f}^{*}\right)$ be two fs-topological spaces where

$$
\begin{aligned}
& \tau_{f}=\left\{\Phi, U_{E}^{\sim}, f_{A}=\left\{e_{1}=\left\{x_{0.3}, y_{0.8}, z_{0.5}\right\}, e_{2}=\left\{x_{0.7}, y_{0.8}, z_{0.4}\right\}\right\},\right. \\
& g_{B}=\left\{e_{2}=\left\{x_{0.3}, y_{0.2}, z_{0.8}\right\}, e_{3}=\left\{x_{0.4}, y_{0.6}, z_{0.5}\right\}\right\}, h_{C}=\left\{e_{2}=\left\{x_{0.3}, y_{0.2}, z_{0.4}\right\}\right\}, \\
& s_{E}=\left\{e_{1}=\left\{x_{0.3}, y_{0.8}, z_{0.5}\right\}, e_{2}=\left\{x_{0.7}, y_{0.8}, z_{0.8}\right\},\left\{e_{3}=\left\{x_{0.4}, y_{0.6}, z_{0.5}\right\}\right\} .\right. \\
& \tau_{f}^{*}=\left\{\Phi, V_{P}^{\sim}, k_{D}=\left\{p_{1}=\left\{a_{0.5}, b_{0.7}, c_{0.8}\right\}\right\}, m_{F}=\left\{p_{2}=\left\{a_{0.3}, b_{0.4}, c_{0.8}\right\}\right\},\right. \\
& n_{P}=\left\{p_{1}=\left\{a_{0.5}, b_{0.7}, c_{0.8}\right\},\left\{p_{2}=\left\{a_{0.3}, b_{0.4}, c_{0.8}\right\}\right\} .\right.
\end{aligned}
$$

Let $\varphi: U \rightarrow V, \psi: E \rightarrow P$ be two mappings where $\varphi(x)=\varphi(y)=a, \varphi(z)=b, \psi\left(e_{1}\right)=$ $p_{2}, \psi\left(e_{2}\right)=p_{1}$. Then the fs-mapping $\varphi_{\psi}$ is is fs- $\tau$-continuous at $e_{2 x}{ }^{0.3}$.

\section{Fuzzy Soft Cotopological Spaces}

Definition 17. Let $U$ be the universe and $E$ be the parameter set. A fs-cotopological space is a pair $\left(U_{E}^{\sim}, \kappa_{f}\right)$ where $\kappa_{f}$ is a family of fs-sets over $U$ which holds the following conditions:

i) $\Phi, U_{E}^{\sim} \in \kappa_{f}$, ii) If $k_{A}, m_{B} \in \kappa_{f}$ then $k_{A} \sqcup m_{B} \in \kappa_{f}$,

iii) If $\left(k_{A}\right)_{i \in I} \in \kappa_{f}$ then $\Pi_{i \in I}\left(k_{A}\right)_{i \in I} \in \kappa_{f}$.

$\kappa_{f}$ is called a fs-cotopology of fs-sets over $\mathrm{U}$. The pair $\left(U_{E}^{\sim}, \kappa_{f}\right)$ is called fs-cotopological space. The elements of $\kappa_{f}$ are called fs-closed sets.

Definition 18. Let $r_{A}$ be a fs-set over U. $r_{A}$ is calledthe fs-remote neighborhood of $e_{x}^{\lambda}$ if there exists a fs-closed set $k_{B}$ such that $e_{x}^{\lambda} \notin^{\sim} k_{B} \sqsupseteq r_{A}$. The family of all fs-remote neighborhhods of $e_{x}^{\lambda}$ is denoted by $\mathcal{R} \aleph\left(e_{x}^{\lambda}\right)$.

Example 3. Let $E=\left\{e_{1}, e_{2}, e_{3}\right\}, U=\{x, y\}$ and

$\kappa_{f}=\left\{\Phi, U_{E}^{\sim}, k_{A}=\left\{e_{1}=\left\{x_{0.3}, y_{0.5}, z_{0.7}\right\}, e_{2}=\left\{x_{0.7}, y_{0.6}, z_{0.3}\right\}\right\}\right.$,

$m_{B}=\left\{e_{1}=\left\{x_{0.2}, y_{0.3}, z_{0.8}\right\}, \quad e_{3}=\left\{x_{0.9}, y_{0.3}, z_{0.6}\right\}\right\}, n_{C}=\left\{e_{1}=\left\{x_{0.2}, y_{0.3}, z_{0.7}\right\}\right\}$,

$s_{E}=\left\{e_{1}=\left\{x_{0.3}, y_{0.5}, z_{0.8}\right\}, e_{2}=\left\{x_{0.7}, y_{0.6}, z_{0.3}\right\}, e_{3}=\left\{x_{0.9}, y_{0.3}, z_{0.6}\right\}\right\}$

Then $r_{C}=\left\{e_{1}=\left\{x_{0.1}, y_{0.2}, z_{0.5}\right\}\right\} \in \mathcal{R} \aleph\left(e_{1_{x}}^{0.5}\right)$ since $e_{1_{x}}^{0.5} \notin^{\sim} m_{B} \sqsupseteq r_{C}$.

Theorem 6. Let $\left(U_{E}^{\sim}, \kappa_{f}\right)$ be a fs- two fs-sets over U. Then the followings hold cotopological space, $e_{x}^{\lambda}$ be a fs-point, $r_{A}, s_{B}$ be for $\mathcal{R} \aleph\left(e_{x}^{\lambda}\right)$ : 
i) If $r_{A} \in \mathcal{R} N\left(e_{x}^{\lambda}\right)$ then $e_{x}^{\lambda} \notin^{\sim} r_{A}$.

ii) If $r_{A}, s_{B} \in \mathcal{R} N\left(e_{x}^{\lambda}\right)$ then $r_{A} \sqcup s_{B} \in$ $\mathcal{R} \aleph\left(e_{x}^{\lambda}\right)$.

iii) If $r_{A} \sqsubseteq s_{B}$ and $s_{B} \in \mathcal{R} \aleph\left(e_{x}^{\lambda}\right)$ then $r_{A} \in$ $\mathcal{R} \aleph\left(e_{x}^{\lambda}\right)$.

Proof. 1. It is clear from the definition 18 .

2. Let $r_{A}, s_{B} \in \mathcal{R} \aleph\left(e_{x}^{\lambda}\right)$. Then there exist fsclosed sets $k_{A_{1}}, m_{B_{1}}$ such that

$e_{x}^{\lambda} \notin^{\sim} k_{A_{1}} \sqsupseteq r_{A}$ and $e_{x}^{\lambda} \notin^{\sim} m_{B_{1}} \sqsupseteq s_{B}$.

It is seen that $\lambda>k_{A_{1}}{ }^{e}(x)>r_{A}{ }^{e}(x)$ and $\lambda>m_{B_{1}}{ }^{e}(x)>s_{B}{ }^{e}(x)$ and hence $\lambda>\max \left\{k_{A_{1}}{ }^{e}(x), m_{B_{1}}{ }^{e}(x)\right\}>$ $\max \left\{r_{A}^{e}(x), s_{B}^{e}(x)\right\}$.

This shows that $r_{A} \sqcup s_{B} \in \mathcal{R} \aleph\left(e_{x}^{\lambda}\right)$.
3. Let $s_{B} \in \mathcal{R} N\left(e_{x}^{\lambda}\right)$. Then there exists a fsclosed set $m_{B_{1}}$ such that $e_{x}^{\lambda} \notin^{\sim} m_{B_{1}} \sqsupseteq s_{B}$. Since $r_{A} \sqsubseteq s_{B}, e_{x}^{\lambda} \notin^{\sim} m_{B_{1}} \sqsupseteq r_{A}$. It shows that $r_{A} \in \mathcal{R} \aleph\left(e_{x}^{\lambda}\right)$.

Theorem 7. Let $\left(U_{E}^{\sim}, \kappa_{f}\right)$ be a fscotopological space, $e_{x}^{\lambda}$ be a fs-point. The family of all sets of the fs-remote neighborhood of $e_{x}^{\lambda}$ is a $\sigma$-ideal, i.e,is hereditary and additive.

Proof. The proof is obvious from Theorem 6 .

Definition 19. Let $\left(U_{E}^{\sim}, \kappa_{f}\right)$ be a fscotopological space, $f_{A}$ be a fs-set over $\mathrm{U}$. The intersection of all fs-closed sets containing $f_{A}$ is called the closure of $f_{A}$ and is denoted by $c l f_{A}$.

$$
c l f_{A}=\prod_{i \in I}\left\{v_{B_{i}} \sqsubseteq U_{E}^{\sim}: v_{B_{i}} \in \kappa_{f}, f_{A} \sqsubseteq v_{B_{i}}, i \in I\right\}
$$

Theorem 8. Let $\left(U_{E}^{\sim}, \kappa_{f}\right)$ be a fs- i)If $v_{A}$ is a fs-Q-coneighborhood of $e_{x}^{\lambda}$ then cotopological space and $f_{A}$ be a fs-set over $\mathrm{U} . \quad e_{x}^{1-\lambda}$ is not quasi coincident with $v_{A}$.

The followings hold:

i) $c l f_{A}$ is a fs-closed set.

ii) $f_{A} \sqsubseteq c l f_{A}$.

iii) $c l f_{A}$ is the smallest fs-closed set containing $f_{A}$.

iv) $f_{A} \in \kappa_{f}$ iff $f_{A}=c l f_{A}$.

Proof. The proof can be done similarly to the analogous statements Simsekler and Yuksel (2013).

Definition 20. Let $\left(U_{E}^{\sim}, \kappa_{f}\right)$ be a fscotopological space $v_{A}$ be a fs-set over U. $v_{A}$ is called fs-Q-coneighborhood of $e_{x}^{\lambda}$ if there exists a fs-closed set $k_{B}$ such that $e_{x}^{1-\lambda}$ is not quasi coincident with $k_{B}$ and $k_{B} \sqsupseteq v_{A}$.

ii) If $v_{A}, w_{B}$ are fs-Q-coneighborhood of $e_{x}^{\lambda}$ then $v_{A} \sqcup w_{B}$ is a fs-Q-coneighborhood of $e_{x}^{\lambda}$.

iii) If $v_{A}$ is a fs-Q-coneighborhood of $e_{x}^{\lambda}$ and $w_{B} \subseteq v_{A}$ then $w_{B}$ is a fuzzuy soft Qconeighborhood of $e_{x}^{\lambda}$.

Definition 21. Let $\left(U_{E}^{\sim}, \kappa_{f}\right),\left(V_{P}^{\sim}, \kappa_{f}^{*}\right)$ be two fs-topological spaces, $\varphi: U \rightarrow V, \psi: E \rightarrow P$ be mappings and $e_{x}^{\lambda}$ be a fs-point. $\varphi_{\psi}=$ $(\varphi, \psi): F S(U, E) \rightarrow F S(V, P)$ is called fs- $\kappa$ continuous at $e_{x}^{\lambda}$ if for any fs-remote neighborhood $v_{A}$ of $\varphi_{\psi}\left(e_{x}^{\lambda}\right)$, there exists a fsremote neighborhood $w_{B}$ of $e_{x}^{\lambda}$ such that $\varphi_{\psi}\left(w_{B}\right) \sqsubseteq v_{A}$.

Theorem 9. Let $\left(U_{E}^{\sim}, \kappa_{f}\right)$ be a fscotopological space and $v_{A}, w_{B}$ be two fs-sets over U. Then the followings hold:

Theorem 10. Let $\left(U_{E}^{\sim}, \kappa_{f}\right),\left(V_{P}^{\sim}, \kappa_{f}^{*}\right)$ be two fs-topological spaces, $\varphi: U \rightarrow V, \psi: E \rightarrow P$ be mappings, $e_{x}^{\lambda}$ be a fs-point and $\varphi_{\psi}=$ 
$(\varphi, \psi): F S(U, E) \rightarrow F S(V, P)$ be a mapping. Then the followings are equivalent:

i) $\varphi_{\psi}=(\varphi, \psi): F S(U, E) \rightarrow F S(V, P)$ is fs- $\kappa$ continuous mapping at $e_{x}^{\lambda}$.

ii) For any fs-remote neighborhood $v_{A}$ of $\varphi_{\psi}\left(e_{x}^{\lambda}\right)$ there exists a fs-remote neighborhood $w_{B}$ of $e_{x}^{\lambda}$ such that $w_{B} \sqsupseteq$ $\varphi_{\psi}{ }^{-1}\left(v_{A}\right)$.

iii) The inverse image of every fs-remote neighborhood of $\varphi_{\psi}\left(e_{x}^{\lambda}\right)$ is a fs-remote neighborhood of $e_{x}^{\lambda}$.

\section{Fuzzy Soft Ditopological Spaces}

After the definitions and theorems mentioned in the previous sections we can give the main purpose of this paper- a fs-ditopological space, which is a synthesis of a fs-topology, connected to the property of opennes in the space and a fs-cotopology, relaying on the property of closedness in the space.

Definition 22. The triple $\left(U_{E}^{\sim}, \tau_{f}, \kappa_{f}\right)$ is said to be a fs-ditopological space if $U_{E}^{\sim}$ is a fs-set, $\tau_{f}$ is a fs-topology and $\kappa_{f}$ is a fs-cotopology on $U_{E}^{\sim}$. A pair $\delta=\left(\tau_{f}, \kappa_{f}\right)$ is called a fsditopology on $U_{E}^{\sim}$.

Definition 23. Let $\left(U_{E}^{\sim}, \delta\right)$ be a fsditopological space, $f_{B}, m_{C}$ be two fs-sets on $\mathrm{U}$ and $e_{x}^{\lambda}$ be a fs-point. A pair $\left(f_{B}, m_{C}\right)$ is called a fs-neighborhood of $e_{x}^{\lambda}$ if $f_{B}$ is a fs- $\tau$ neighborhood and $m_{C}$ is a fs-remote neighborhood of $e_{x}^{\lambda}$.

Fs-interior and fs-closure of a fs-set $f_{A}$ in a fsditopological space $\left(U_{E}^{\sim}, \delta\right)$ are defined respectively by:

$$
\begin{gathered}
\operatorname{int}_{A}=\bigsqcup_{i \in I}\left\{g_{B_{i}} \sqsubseteq U_{E}^{\sim}: g_{B_{i}} \in \tau_{f}, g_{B_{i}} \sqsubseteq f_{A}, i \in I\right\} \\
c l f_{A}=\prod_{i \in I}\left\{v_{B_{i}} \sqsubseteq U_{E}^{\sim}: v_{B_{i}} \in \kappa_{f}, f_{A} \sqsubseteq v_{B_{i}}, i \in I\right\}
\end{gathered}
$$

Definition 24. Let $\left(U_{E}^{\sim}, \delta_{1}\right),\left(V_{P}^{\sim}, \delta_{2}\right)$ be two fs-ditopological spaces. A mapping $\varphi_{\psi}=(\varphi, \psi):\left(U_{E}^{\sim}, \delta_{1}\right) \rightarrow\left(V_{P}^{\sim}, \delta_{2}\right)$ is called fs-continuous if the preimage of any fs-set from $\tau_{2}$ is in $\tau_{1}$ and the preimage of any fsset from $\kappa_{2}$ is in $\kappa_{1}$.

Theorem 11. A mapping

$\varphi_{\psi}=(\varphi, \psi):\left(U_{E}^{\sim}, \delta_{1}\right) \rightarrow\left(V_{P}^{\sim}, \delta_{2}\right) \quad$ be a mapping Then the followings are equivalent:

i) $\varphi_{\psi}$ is fs-continuous at $e_{x}^{\lambda}$.

ii) For any fs- $\tau$ - neighborhood $f_{A}$ and fsremote neighborhood $v_{A}$ of $\varphi_{\psi}\left(e_{x}^{\lambda}\right)$ there exist a fs- $\tau$ - neighborhood $g_{B}$ and a fs-remote neighborhood $w_{B}$ of $e_{x}^{\lambda}$ such that $g_{B}$ 드 $\varphi_{\psi}{ }^{-1}\left(f_{A}\right)$ and $w_{B} \sqsupseteq \varphi_{\psi}{ }^{-1}\left(v_{A}\right)$ respectively. iii) The preimage of every fs- $\tau$ - neighborhood of $\varphi_{\psi}\left(e_{x}^{\lambda}\right)$ is a fs- $\tau$ - neighborhood of $e_{x}^{\lambda}$ and the preimage of every fs-remote neighborhood of $\varphi_{\psi}\left(e_{x}^{\lambda}\right)$ is a fs-remote neighborhood of $e_{x}^{\lambda}$.

Proof. The proof is obvious by Theorem 5 . and Theorem 10.

\section{References}

Ahmad, B. and Hussain, S., 2012. On some structures of soft topology. Mathematical Sciences, DOI: 10.1186/2251-7456-6-64.

Aktas, H. and Cagman, N. 2007. Soft sets and soft groups. Inform. Sci., 77, 2726-2735. 
Atmaca, S. and Zorlutuna, I. 2013. On fuzzy soft topological spaces. Annals of Fuzzy Mathematics and Informatics, 5(2), 377-386.

Atmaca, S. and Zorlutuna, I. 2014. On Topological Structures of Fuzzy Parametrized Soft Sets. The Scientific World Journal, Article ID164176.

Aygunoglu, A. and Aygun, H. 2011. Some notes on soft topological spaces. Neural Computing and Applications, 21(1),113-119.

Aygunoglu, A., Cetkin, V. and Aygun, H. 2014. An introduction to fs-topological spaces. Hacettepe Journal of Mathematics and Statistics,43(2),197-208.

Bera, S, Roy, S.K, Karaaslan, F, and Cağman, N. 2017. Soft congruence relation over lattices, Hacettepe Journal of Mathematics and Statistics, 46(6), 1035 - 1042.

Brown, L.M. and Diker, M., 1998. Ditopological texture spaces and intuitionistic sets. Fuzzy Sets and Systems, 98, 217-224.

Cagman, N., Karatas, S., and Enginoglu, S. 2011. Soft topology. Computers and Mathematics with Applications, 62, 351-358.

Chang, C.L.1968. Fuzzy topological spaces. J. Math. Appl., 24, 182-193.

Feng, F., Jun, Y.B. and Zhao, X. 2008. Soft semirings. Computers and Mathematics with Applications, 56, 2621-2628.

Karaaslan, F, Cagman, N, and Enginoglu, S. 2012. Soft Lattices, Journal of New Results in Science (1), 5-17.

Karaaslan, F. 2016. Soft Classes and Soft Rough Classes with Applications in Decision Making. Mathematical Problems in
Engineering, $1-11$.

Doi: $10.1155 / 2016 / 1584528$.

Karaaslan, F, Çagman N. 2013. Fuzzy Soft Lattice Theory, ARPN Journal of Science and Technology, 3(3).

Kelly, J.L. 1963. Bitopological spaces. Proc. Lond. Math.Soc. III Ser, 13, 71-89.

Maji, P.K. Biswas, R. and Roy, A.R. 2001. Fuzzy soft sets. J. Fuzzy Math. 9(3), 589-602.

Min, W.K. 2011. A note on soft topological spaces. Comput. Math. Appl., 62, 3524-3528.

Molodtsov, D. 1999. Soft set theory-First results. Computers Math. Appl.,37(4/5), 1931.

Nazmul, S. and Samanta, S.K. 2010. Soft topological groups. Kochi J. Math., 5,151161.

Ozturk, T. 2016. A new approach to soft uniform spaces,Turkish Journal of Mathematics, 40, 1071-1084.

Pazar Varol, B. and Aygun, H. 2012. Fuzzy soft topology. Hacettepe Journal of Mathematics and Statistics, 41(3), 407-419.

Roy, S. and Samanta, T.K. 2012. A note on fuzzy soft topological spaces. Annals of Fuzzy Mathematics and Informatics, 3, 305311.

Roy, S. and Samanta, T.K. 2013. An introduction to open and closed sets on fuzzy soft topological spaces. Annals of Fuzzy Math. and Inf., 6(2), 425-431.

Shabir, M. and Naz, M. 2011. On soft topological spaces. Comput. Math. Appl.,61, 1786-1799. 
Simsekler, T. and Yuksel, S. 2013. Fuzzy soft topological spaces. Annals of Fuzzy Mathematics and Informatics, 5(1), 87-96.

Simsekler Dizman, T., Sostak, A. and Yuksel, S. 2016. Soft Ditopological Spaces. Filomat, 30(1), 209-222.

Tanay, B. and Kandemir, M.B. 2011. Topological structures of fuzzy soft sets. Computers and Mathematics with Applications, 61, 412-418.

Zadeh, L.A. 1965. Fuzzy sets. Information and Control, 8, 338-353.

Zorlutuna, I, Akdag, M., Min, W.K. and Atmaca, S. 2012. Remarks on soft topological spaces. Annals of Fuzzy Mathematics and Informatics, 3(2), 171-185. 